\title{
TAUTNESS AND LOCALLY FINITELY-VALUED ALEXANDER-SPANIER COCHAINS
}

\author{
SATYA DEO AND ATUL N. ROY
}

(Communicated by Haynes R. Miller)

\begin{abstract}
In this paper we prove that the following two subspaces are tautly embedded with respect to Alexander-Spanier cohomology based on locally finitely-valued cochains: (i) any neighborhood retract of arbitrary topological space, and (ii) any compact subset of a Hausdorff space.
\end{abstract}

1. Introduction and preliminaries. The importance of Alexander-Spanier cohomology groups $H_{L}^{*}(X)$ of a space $X$ based on locally finitely-valued cochains has been sufficiently explained by Massey (cf. [WM, p. 225]). He has presented, as far as possible, a systematic development of these groups side by side along with the classical Alexander-Spanier cohomology groups $H^{*}(X)$ based on all cochains. If $X$ is a paracompact Hausdorff space, then Gordon's Isomorphism Theorem states that the two groups $H^{*}(X)$ and $H_{L}^{*}(X)$ are naturally isomorphic (ibid p. 232).

Among many interesting properties, Massey has proved (ibid p. 242) some tautness results for Alexander-Spanier cohomology based on locally finitely-valued cochains. Precisely, he proves that the following subspaces are tautly embedded with respect to Alexander-Spanier cohomology based on locally finitely valued cochains: (1) a closed subspace of a paracompact Hausdorff space, and (ii) any subspace of a completely paracompact space. The proof of these results, however, follows directly from Gordon's Isomorphism Theorem stated above and the following theorem (cf. [ES]):

(1.1) THEOREM. The following subspaces are tautly embedded with respect to Alexander-Spanier cohomology based on all cochains:

(i) a closed subspace of a paracompact space (Spanier),

(ii) any subspace of a completely paracompact space (Sitnikov),

(iii) a compact subspace of a Hausdorff (Bredon), and

(iv) a neighborhood retract of an arbitrary space (Deo).

The question whether or not the results analogous to (iii) and (iv) above are true has not been answered for Alexander-Spanier cohomology based on locally finitely-valued cochains.

The main objective of this paper is to answer this question in the affirmative. Our approach essentially consists of obtaining required modifications so that the method of proof, known for the case of groups $H^{*}(X)$, can be applied for the groups

Received by the editors November 26, 1986.

1980 Mathematics Subject Classification (1985 Revision). Primary 55B05, 55B99; Secondary $55 \mathrm{~B} 10$.

Key words and phrases. Locally zero and locally finitely-valued cochains, Alexander-Spanier cohomology, tautness. 
$H_{L}^{*}(X)$ as well, at least in the two unknown cases corresponding to (iii) and (iv) above.

For a given topological space $X$ and any coefficient group $G$, let $C^{p}(X ; G)$ denote the group of all functions $\varphi: X^{p+1} \rightarrow G$ with pointwise operations. Such a function $\varphi$ is said to be locally zero if there exists an open covering $\mathcal{U}$ of $X$ such that whenever $x_{0}, x_{1}, \ldots, x_{p} \in U$ for some $U \in U$, then $\varphi\left(x_{0}, x_{1}, \ldots, x_{p}\right)=0$; we normally abbreviate this fact by saying that $\varphi$ vanishes on $\mathcal{U}^{p+1}$. A function $\varphi: X^{p+1} \rightarrow G$ is said to be locally finite-valued if each $(p+1)$-tuple $\left(x_{0}, x_{1}, \ldots, x_{p}\right)$ has a neighborhood $N$ in $X^{p+1}$ such that $\varphi \mid N$ takes on only finitely many values in $G$. The group of all locally zero functions $\varphi$ on $X^{p+1}$ is denoted by $C_{0}^{p}(X ; G)$ and the group of all locally finitely-valued functions on $X^{p+1}$ is denoted by $C_{L}^{p}(X ; G)$. The cohomology groups $H^{*}(X ; G)$ of the quotient cochain complex $C^{*}(X ; G) / C_{0}^{*}(X ; G)$ are called the Alexander-Spanier cohomology groups of $X$ based on all cochains. The cohomology groups $H_{L}^{*}(X ; G)$ of the quotient cochain complex $C_{L}^{*}(X ; G) / C_{L}^{*}(X ; G) \cap C_{0}^{*}(X ; G)$ are called Alexander-Spanier cohomology based on all locally finitely-valued cochains.

A neighborhood means an arbitrary neighborhood, not necessarily open unless stated otherwise. If $A$ is a subspace of $X$, then by an open cover $\mathcal{U}$ of $A$ in $X$ we mean a collection of open sets of $X$ whose union covers $A$. All our notations and terminology are standard, but to avoid any confusion we will specifically follow [S].

The author expresses his thanks to the referee for his suggestions which, in particular, improved the proof of Theorem (2.7).

2. Main result. Recall that if $A$ is a subspace of a topological space $X$, then the inclusion maps $A \subseteq U$, where $U$ runs over all neighborhoods of $A$ in $X$, define a canonical homomorphism

$$
\eta: \lim _{\longrightarrow} H_{L}^{p}(U ; G) \rightarrow H_{L}^{p}(A ; G) .
$$

Following [S, p. 289], the subspace $A$ is said to be taut in $X$ with respect to Alexander-Spanier cohomology based on locally finitely-valued cochains if the homomorphism $\eta$ is an isomorphism for each $p \geq 0$ and every coefficient group $G$. Hence onwards we will suppress the coefficient group $G$ from our notations $H_{L}^{p}(A ; G)$, etc. Let us now prove the following

(2.2) THEOREM. Let $A$ be a neighborhood retract of a space $X$. Then $A$ is tautly embedded in $X$ with respect to Alexander-Spanier cohomology $H_{L}^{*}$ based on locally finitely-valued cochains.

ProOF. Let $N$ be a neighborhood of $A$ in $X$ and let $r: N \rightarrow A$ be a retraction. If $\alpha \in H_{L}^{p}(A)$, then because $r$ is continuous, $r^{*}(\alpha) \in H_{L}^{p}(N)$. Also since $r \mid A$ is identity on $A, r^{*}(\alpha) \mid A=\alpha$. This means $\eta$ of (2.1) is an epimorphism.

Next, suppose $\alpha \in H_{L}^{p}(M)$ for some neighborhood $M$ of $A$ in $N$ such that $\alpha \mid A=0 \in H_{L}^{p}(A)$. Let $\varphi \in C_{L}^{p}(M)$ be a cochain representing $\alpha$. This means there exists an open covering $\mathcal{U}_{1}$ of $M$ such that $\delta \varphi=0$ on $U_{1}^{p+2}$. Also, since $\alpha \mid A=0$, there exists an open covering $\mathcal{U}_{2}$ of $A$ in $X$ and $\varphi^{\prime} \in C_{L}^{p-1}(A)$ such that $\delta \varphi^{\prime}=\varphi$ on $\mathcal{U}_{2}^{p+1} \cap A^{p+1}$. Let $\mathcal{U}=\mathcal{U}_{1} \cap \mathcal{U}_{2}$ be the open covering of $A$ in $X$. Then both of the above equations hold when all points belong to some member of $\mathcal{U} \cap A$. Define $\mathcal{V}=\left\{U \cap r^{-1}(U \cap A) \mid U \in \mathcal{U}\right\}$ and put $S=\operatorname{St}(A ; \mathcal{V}), f=r \mid S$. Note that for 
each $V \in \mathcal{V}, V \cup f(V) \subseteq U$ for some $U \in \mathcal{U}$. Let us now define the usual chain deformation homomorphism $D: C_{L}^{p}(S) \rightarrow C_{L}^{p-1}(S)$ by the formula

$$
D \psi\left(x_{0}, \ldots, x_{p-1}\right)=\sum_{i=0}^{p-1}(-1)^{i} \psi\left(x_{0}, \ldots, x_{i}, f\left(x_{i}\right), \ldots, f\left(x_{p-1}\right)\right)
$$

where $\psi \in C_{L}^{p}(S)$. Then one can easily verify that

$$
\delta D \psi=f^{\#}(\psi \mid A)-\psi-D \delta \psi
$$

We replace $\psi$ by $\varphi \mid S$, and note that whenever $\left(x_{0}, \ldots, x_{p}\right)$ are in some member of $\mathcal{V}$, then

$$
(\delta D(\varphi \mid S))\left(x_{0}, \ldots, x_{p}\right)=\left(\delta\left(f^{\#} \varphi^{\prime}\right)\right)\left(x_{0}, \ldots, x_{p}\right)-(\varphi \mid S)\left(x_{0}, \ldots, x_{p}\right) .
$$

Now we proceed to prove that both $\delta\left(f^{\#} \varphi^{\prime}\right), \delta(D(\varphi \mid S)) \in C_{L}^{p}(S)$. For this it suffices to prove the following

(2.5) PROPOSITION. In the above situation,

(i) $\varphi \in C_{L}^{p}(A) \Rightarrow f^{\#} \varphi \in C_{L}^{p}(S)$, and

(ii) $\varphi \in C_{L}^{p}(S) \Rightarrow D \varphi \in C_{L}^{p-1}(S)$.

PROOF. Part (i) follows from the fact that $f=r \mid S$ is a continuous function.

In order to avoid lengthy explanation we shall prove part (ii) when $p=3$; the general case is then only analogous and clear. Once again it requires only the continuity of $f$. Consider the formula

$$
\begin{aligned}
(D \varphi)\left(x_{0}, x_{1}, x_{2}\right)= & \varphi\left(x_{0}, f\left(x_{0}\right), f\left(x_{1}\right), f\left(x_{2}\right)\right)-\varphi\left(x_{0}, x_{1}, f\left(x_{1}\right), f\left(x_{2}\right)\right) \\
& +\varphi\left(x_{0}, x_{1}, x_{2}, f\left(x_{2}\right)\right) .
\end{aligned}
$$

Now $\varphi$ is locally finitely-valued means there are neighborhoods $U_{0} \times U_{1} \times U_{2} \times U_{3}$ of $\left(x_{0}, f\left(x_{0}\right), f\left(x_{1}\right), f\left(x_{2}\right)\right), V_{0} \times V_{1} \times V_{2} \times V_{3}$ of $\left(x_{0}, x_{1}, f\left(x_{1}\right), f\left(x_{2}\right)\right)$ and $W_{0} \times$ $W_{1} \times W_{2} \times W_{3}$ of $\left(x_{0}, x_{1}, x_{2}, f\left(x_{2}\right)\right)$ such that each of the above three terms is finitely-valued on the corresponding product neighborhoods. By continuity of $f$ we can choose neighborhoods

(i) $N_{0}$ of $x_{0}$ so that $N_{0} \subseteq U_{0} \cap V_{0} \cap W_{0}$ and $f\left(N_{0}\right) \subseteq U_{1}$,

(ii) $N_{1}$ and $x_{1}$ such that $N_{1} \subseteq V_{1} \cap W_{1}$ and $f\left(N_{1}\right) \subseteq U_{2} \cap V_{2}$, and

(iii) $N_{2}$ of $x_{2}$ such that $N_{2} \subseteq W_{2}$ and $f\left(N_{2}\right) \subseteq U_{3} \cap V_{3} \cap W_{3}$.

Then $N_{0} \times N_{1} \times N_{2}$ is a neighborhood of $\left(x_{0}, x_{1}, x_{2}\right)$ such that $D \varphi$ is finitelyvalued on this neighborhood. This completes the proof of (2.5).

Now, this proposition combined with (2.4) implies that $\varphi \mid C_{L}^{p}(S)$ is a coboundary in the Alexander-Spanier cochain complex of $S$ based on locally finitely-valued chochains. Hence $\alpha \mid H_{L}^{p}(S)=0$. This means $\eta$ is a monomorphism. Q.E.D.

(2.6) REMARK. If $X$ is a compact space, then a locally finitely-valued cochain is clearly a finitely-valued cochain. Now as in Corollary 3 of [SD], we can apply the above tautness result to prove the homotopy axiom for Alexander-Spanier cohomology based on finitely-valued cochains for compact spaces.

(2.7) THEOREM. Let $A$ be a compact subset of a Hausdorff space $X$. Then $A$ is tautly embedded in $X$ with respect to Alexander-Spanier cohomology $H_{L}^{*}$ based on locally finitely-valued cochains.

ProOF. To prove that $\eta$ of $(2.1)$ is onto, let $\alpha \in H_{L}^{p}(A)$. Choose a $\varphi \in C_{L}^{p}(A)$ representing $\alpha$. Then $\delta \varphi$ is locally zero and so there is an open cover $\mathcal{U}$ of $A$ in 
$X$ such that $\delta \varphi$ vanishes on $\mathcal{U}^{p+2} \cap A^{p+2}$. Since $\varphi$ is locally finitely-valued on $A$ and $A$ is compact, $\varphi$ is actually finitely-valued on $A$. Consider a star-refinement $\{V \cap A \mid V \in \mathcal{V}\}$ of $\{U \cap A \mid U \in \mathcal{U}\}$ and define a function $f: W=\operatorname{St}(A ; \mathcal{V}) \rightarrow A$ such that $f \mid A$ is the identity on $A$ and for each $V \in \mathcal{V}, V \cup f(V) \subseteq U$ for some $U \in \mathcal{U}$.

Now it is plain that $f^{\#} \varphi$ is finitely-valued on $W$ because $\varphi$ is finitely-valued on $A$. Next observe that

$$
\delta\left(f^{\#} \varphi\right)=f^{\#}(\delta \varphi)
$$

and so if all points $x_{0}, \ldots, x_{p+1}$ lie in some member of $\mathcal{V}$, then $f\left(x_{0}\right), \ldots, f\left(x_{p+1}\right)$ are all in some member of $\mathcal{U}$. Consequently, $\delta\left(f^{\#} \varphi\right)$ vanishes on $\mathcal{V}^{p+2} \cap W^{p+2}$. This implies that $f^{\#} \varphi$ is a cocycle. Now if $\alpha^{\prime} \in H_{L}^{p}(W)$ is represented by this cocycle, then $\alpha^{\prime} \mid A=\alpha$.

To prove that $\eta$ is a monomorphism, let $N$ be an open neighborhood of $A$ and $\alpha \in H_{L}^{p}(N)$ such that $\alpha \mid A=0 \in H_{L}^{p}(A)$. Choose a cochain $\varphi \in C_{L}^{p}(N)$ which represents $\alpha$. Then there is an open covering $\mathcal{U}_{1}$ of $N$ such that $\delta \varphi$ vanishes on $\mathcal{U}_{1}^{p+2} \cap N^{p+2}$. Also, since $\varphi \mid A$ represents the zero cohomology class, there exists another open covering $\mathcal{U}_{2}$ of $A$ in $X$ and a $\varphi^{\prime} \in C_{L}^{p-1}(A)$ such that $\varphi-\delta \varphi^{\prime}$ vanishes on $\mathcal{U}_{2}^{p+1} \cap A^{p+1}$. Let $\mathcal{U}=\left\{U_{1} \cap U_{2} \mid U_{1} \in \mathcal{U}_{1}, U_{2} \in \mathcal{U}_{2}\right\}$, and apply Spanier's Lemma [ES] to this cover $U$ to get another open cover $\mathcal{V}$ of $A$ in $X$ and a function $f: S=\operatorname{St}(A ; \mathcal{V}) \rightarrow A$ such that $f \mid A$ is the identity and for each $V \in \mathcal{V}$, $V \cup f(V) \subseteq U$ for some $U \in U$. As noted earlier, $f^{\#}(\varphi)$ is finitely-valued and so is in $C_{L}^{p}(S)$. We next claim that $D \varphi \in C_{L}^{p-1}(S)$. To simplify matters, once again we examine the case $p=3$ and refer to the formula written in the proof of Proposition (2.5). The following fact can be easily verified: If $A$ is a compact space and $\varphi \in X \times A$ is a locally finitely-valued function, then for each $x \in X$, there exists a neighborhood $U$ of $x$ such that $\varphi \mid U \times A$ is actually finitely-valued. Let $\left(x_{0}, x_{1}, x_{2}\right) \in S^{3}$. The map $\left(x_{0}, f\left(x_{0}\right), f\left(x_{1}\right), f\left(x_{2}\right)\right) \rightarrow \varphi\left(x_{0}, f\left(x_{0}\right), f\left(x_{1}\right), f\left(x_{2}\right)\right)$ is the restriction to $S \times A \times A \times A$ of a locally finitely-valued function $\varphi \in C_{L}^{3}(S)$ and so, by the above fact, there exists a neighborhood $U_{0}$ of $x_{0}$ such that the above function is finitely-valued on $U_{0} \times A \times A \times A$. Similarly, $\left(x_{0}, x_{1}, f\left(x_{1}\right), f\left(x_{2}\right)\right) \rightarrow$ $\varphi\left(x_{0}, x_{1}, f\left(x_{1}\right), f\left(x_{2}\right)\right)$ is the restriction of $\varphi \in C_{L}^{3}(S)$ to $S \times S \times A \times A$ and so there is a neighborhood $V_{0} \times V_{1}$ of $\left(x_{0}, x_{1}\right) \in S \times S$ such that $\varphi$ is finitelyvalued on $V_{0} \times V_{1} \times A \times A$. Likewise, there is a neighborhood $W_{0} \times W_{1} \times W_{2}$ of $\left(x_{0}, x_{1}, x_{2}\right)$ such that $\varphi$ is finitely-valued on $W_{0} \times W_{1} \times W_{2} \times A$. Now put $N_{0}=U_{0} \cap V_{0} \cap W_{0}, N_{1}=U_{1} \cap W_{1}$ and $N_{2}=W_{2}$. Then $N_{0} \times N_{1} \times N_{2}$ is a neighborhood of $\left(x_{0}, x_{1}, x_{2}\right)$ on which $D \varphi$ is finitely-valued. This establishes our claim, and now the proof follows as in Theorem (2.2). Q.E.D.

(2.8) REMARK. We conclude by recalling that all the four tautness results are now proved for Alexander-Spanier cohomology (a) based on all cochains, (b) based on all locally finitely-valued cochains, and for (c) Alexander-Spanier cohomology with compact supports also. For this last kind of cohomology group, it has been proved in [WM, p. 163] that (i) a closed subspace of locally compact Hausdorff space is tautly embedded. The result analogous to (ii) does not make much sense because these groups are defined only for the category of locally compact Hausdorff spaces and proper continuous maps and an arbitrary subset of such a space need not be locally compact. Finally, the results analogous to (iii) and (iv) are special cases of (i) because compact subsets and retracts of Hausdorff spaces are always closed. 
(2.9) REMARK. It must be observed about the proof of Theorem (2.2) that the crucial point in proving monomorphism as well as epimorphism of $\eta$ was the fact that $f$ was a continuous map. On the other hand, the crucial point in proving $\eta$ to be a monomorphism as well as an epimorphism in Theorem (2.7) was that $A$ is compact. While making these observations, one naturally comes across the following question: Can we give a direct proof (i.e., without using Gordon's Isomorphism Theorem) of the fact that a closed subset of a paracompact space is taut with respect to Alexander-Spanier cohomology based on locally finitely-valued cochains? Our attempts always led us to a proof which could not be termed as distinct from the proof of Gordon's theorem. It will, of course, be interesting to find a neat and clearly different proof of this result so that one could use it and something more to give yet another and equally efficient proof of Gordon's Isomorphism Theorem itself.

\section{REFERENCES}

[SD] Satya Deo, On the tautness property of Alexander-Spanier cohomology, Proc. Amer. Math. Soc. 52 (1975), 441-444.

[WM] W. S. Massey, Homology and cohomology theory, Marcel Dekker, New York, 1978.

[KS] K. Sitnikov, Combinatorial topology of nonclosed sets. I, Amer. Math. Soc. Transl. 15 (1960), 245-295.

[ES] E. H. Spanier, Tautness for Alexander-Spanier cohomology, Pacific J. Math. 75 (1978), 561563.

[S] Algebraic topology, McGraw-Hill, New York, 1966.

DePaRtMent of MAThematics, UNIVERSity of JAMMU, JAMMU-180 001, India (Current address of Satya Deo) INDIA

Department of Mathematics, University of Allahabad, Allahabad-211002,

Current address (A. N. Roy): Department of Mathematics, Rutgers University, New Brunswick, New Jersey 08903 